\title{
A novel feedback active noise control for broadband chaotic noise and random noise
}

\author{
Lei Luo, Jinwei Sun, Boyan Huang
}

\begin{abstract}
The feedback active noise control (ANC) can be seen as a predictor, the conventional method based on filtered$\mathrm{x}$ least mean square (FXLMS) algorithm can only be useful for linear and tonal noise, but for nonlinear and broadband noise, it is useless. The feedback ANC using functional link artificial neural networks (FLANN) based on filtered-s least mean square (FSLMS) algorithm can reduce some nonlinear noise such as chaotic noise, but the noise cancellation performance is not very well, at the same time, it is not useful to random noise. To solve the problem above, a new feedback ANC using wavelet packet FXLMS (WPFXLMS) algorithm is proposed in this paper. By decomposing the broadband noise into several band-limited parts which are predictable and each part is controlled independently, the proposed algorithm can not only suppress the chaotic noise, but also migrate the random noise. Compared with FXLMS and FSLMS algorithms, proposed WPFXLMS algorithm also holds the best performance on noise cancellation. Numerous simulations are conducted to demonstrate the effectiveness of the proposed WPFXLMS algorithm.
\end{abstract}

Keywords: Feedback ANC, Chaotic noise, Random noise, Wavelet packet, FXLMS

\section{Introduction}

In recent years, with the development of industry, excessive noises are produced and seriously impact on people's life and work. Traditional methods to reduce these noise are passive noise control (PNC) using insulation and absorption techniques. However, they can't mitigate the low frequency noise, but most sounds in our life are low frequency signals which are usually less than $1 \mathrm{KHz}$ [1], besides, PNC is also costly and bulky. Compared with PNC, the active noise control (ANC) put forward in the 1970s is an alternative, which can suppress acoustic noise at low frequency with much smaller size and lower cost [2,3]. The ANC system is an electro-acoustic device based on the principle of destructive interference by generating an anti-noise with same amplitude and opposite phase of the undesired noise. Because ANC is an adaptive control system depending on the properties of the noise, it can reduce different noise with the change of environmental conditions, so it is more meaningful in practical applications.

According to the structure of ANC system, it can be classified feedforward ANC and feedback ANC [4]. Comparing with feedforward ANC, the feedback ANC is not involved of reference microphone and only error microphone is required, it reduces the number of system components and cuts back the cost greatly [5-9]. Because there is no reference microphone, so the problem caused by secondary source (loudspeaker) output getting back to the reference microphone and affecting the performance of ANC system can be avoided. Another advantage of feedback ANC is that it is unaffected to multiple noise sources. The principle of feedback ANC is adaptive prediction technology [9-11]. According to the primary noise, the feedback ANC can be subdivided to narrowband feedback ANC and wideband feedback ANC. In this paper, wideband feedback ANC is focused on.

Feedback ANC system has been studied in many paper, most of them are used to cancel linear and tonal noise, which are predictable [5-11]. But for wideband noise like random noise and chaotic noise, less of them are involved. Wideband noise is very common in our life, for example the noise presented by a fan, which shows a combination of tonal noise and chaotic noise [12-15]. Conventional way used in feedback ANC is filtered-x least mean square (FXLMS) algorithm with finite impulse response (FIR) filter or infinite impulse response (IIR) filter, this method 
can only process linear noise and narrowband noise and can't work at all in nonlinear and wideband noise filed. The paper [15] firstly uses functional link artificial neural networks (FLANN) based on filtered-s least mean square (FSLMS) algorithm to reduce chaotic noise in feedback ANC system. To a large extent, it suppresses the chaotic noise, but the performance is not very well. Additionally, it is not involved the random noise. Therefore, for further improving the result of wideband noise cancellation, a better alterative using wavelet packet (WP) is proposed in this paper. Because the secondary path (the acoustic path from speaker to error microphone) shows non-minimum phase response in this paper, it means that the process of this feedback ANC for broadband noise is the process to predict primary noise.

Wavelet transformation has shown promising application in various areas [16,17], it is a main tool to analyze signal in time-frequency domain. By decomposition, the original signal is simplified and presents its local characteristic. Paper [18,19] proposed a wavelet transform domain LMS Algorithm, which has a faster convergence rate than the time domain LMS algorithm. Based on these methods, in paper [20], a multi-resolution FXLMS algorithm using discrete wavelet transform (DWT) is first used in ANC, which present an excellent performance on noise cancellation. But for broadband signal, the DWT can't work well, because it can only make a subdivision for the low frequency part and doesn't have the ability to decompose high frequency part $[21,22]$. While WP method can provide a more careful resolution ratios analysis, since it not only makes a decomposition for the low frequency part of signal, but also makes a decompositions for the high frequency part of signal, this advantage makes WP express an obvious superiority in broadband noise. By analysis above, the feedback ANC using WP for broadband noise would be effective. Through decomposing the broadband noise firstly and reducing each part of the noise independently, the original noise can be cancelled well. After WP decomposition, each part is band-limited, the decomposing level is more, the frequency band is narrower, and each part is more similar to narrowband signal and easier to predict. Because the each part of broadband noise after WP decomposition is controlled by FXLMS algorithm, the proposed algorithm is called WPFXLMS algorithm.

The organization of this paper is as follows. In section 2, some preliminaries are presented, which include the introduction of chaotic noise, discrete wavelet transform and wavelet packet. In section 3, the proposed WPFXLMS algorithm is introduced. Section 4 gives exhaustive simulation studies to confirm the effectiveness of proposed WPFXLMS algorithm. Section 5 concludes the work in this paper.

\section{Preliminaries}

\subsection{Chaotic noise}

The chaotic signals play an important role in signal processing field, we can see them in some deterministic nonlinear system like rotating machines, fans and airfoils etc. Different chaotic signals can be delimited differently, but in term of the natural characteristics of these chaotic signals, they are similar. For example, they are broadband, random-like and difficult to predict the behavior of the systems in long term because of the complexity and sensitive dependence on initial condition of chaotic system. For ANC system, the chaotic signals are seen as an undesired noise to reduce, the most common one is logistic chaotic noise, which can be expressed by

$$
x(n)=\lambda x(n-1)[1-x(n-1)]
$$

where $\lambda=0.4$ and $x(0)=0.9$, and $n$ is the time/sample index.

It is clearly shown from the Eq. (1) that the present state of this noise only depends on its last state. However, in practical cases, the logistic chaotic noise is not always expressed like Eq. (1), the present state of the noise may depend on its past states not the last state. So the logistic chaotic noise can be modified like follow 


$$
x(n)=\lambda x(n-Q)[1-x(n-Q)], \quad\left\{Q \geq 1 \mid Q \in \mathbf{Z}_{+}\right\}
$$

where $\lambda=0.4$ and $\{x(i)\}_{i=0}^{Q-1}=0.9, n=Q, Q+1, \cdots$. In this paper, the chaotic noise like Eq. (2) is called logistic chaotic noise $Q$.

Other than logistic chaotic noise, the Henon chaotic noise is also one common chaotic noise, which can be produced by

$$
\left.\begin{array}{l}
\frac{d y}{d t}=1-a x^{2}+y \\
\frac{d y}{d t}=b x
\end{array}\right\}
$$

where $x$ and $y$ are the chaotic time series, the initial values of $x$ and $y$ are chosen as 0.1 . The parameters are chosen as $a=1.4$ and $b=0.3$.

For comparison, uniform random noise is involved in this paper. The uniform random noise is generated between -1 and 1 by MATLAB function

$$
x(n)=2 \times \text { rand }-1
$$

Referring to the paper $[13,15]$, it can be known that the simplest way to establish the feature of chaotic noise is to plot the present sample with respect to its delayed samples, this plot is usually considered as phase plot. Taking 1000 samples of logistic chaotic noise 1 and random noise to plot their phase plots, which are shown in Fig.1 (a) and (b) respectively. It is seen that the logistic chaotic noise shows dense periodic orbits, which means the points on the orbit are approached randomly. But random signal doesn't display any such periodic orbits.

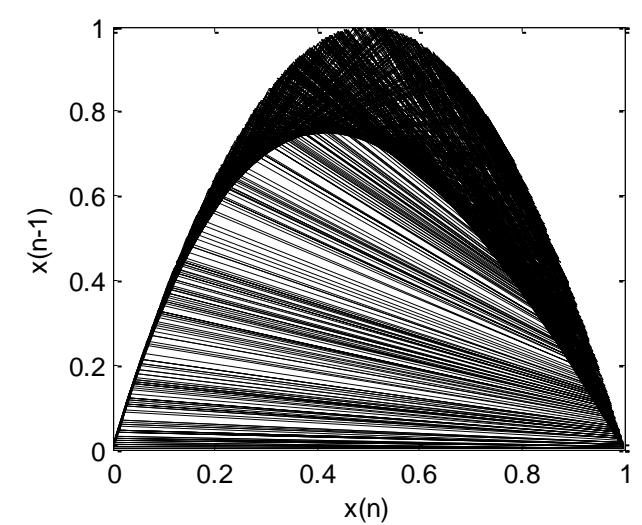

(a) logistic chaotic noise 1

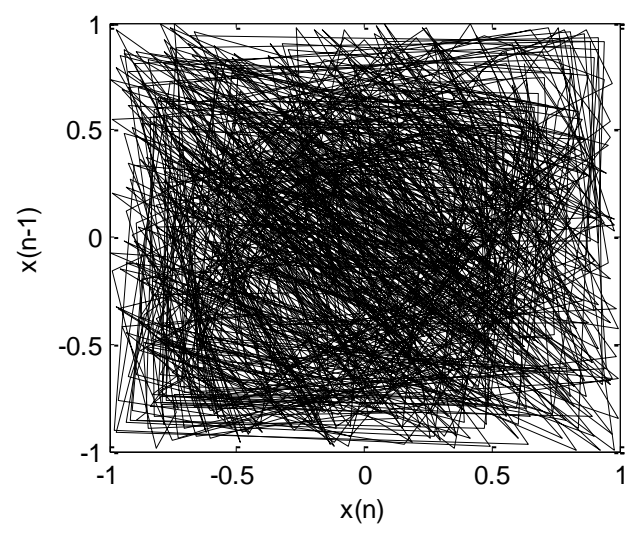

(b) random noise

Fig.1 Phase plots

\subsection{Discrete wavelet transform}

In practical application, especially calculating in computer, the continuous wavelet transform (CWT) must be discretized. Usually, the scale factor $a$ and shift factor $b$ in CWT are replaced by $a=a_{0}^{j}$ and $b=k a_{0}^{j} b_{0}$ $\left(j, k \in \mathbf{Z}, a_{0}>1, b_{0}>0\right)$, so the discrete wavelet function (DWT) can be expressed by

$$
\psi_{j, k}(t)=a_{0}^{-\frac{j}{2}} \psi\left(a_{0}^{-j} t-k b_{0}\right)
$$

In Eq. (5), $a_{0}$ and $b_{0}$ are usually set as $a_{0}=2, b_{0}=1$. Then the discrete wavelet coefficient and reconstructed function are given by 


$$
\begin{gathered}
C_{j, k}=2^{-\frac{j}{2}} \int_{-\infty}^{\infty} f(t) \psi\left(2^{-j} t-k\right) d t \\
f(t)=\sum_{j} \sum_{k} C_{j, k} \psi\left(2^{-j} t-k\right)
\end{gathered}
$$

In the DWT, a signal is represented by inner products with basis functions that are temporal shifts and dilatation of a prototype function (mother wavelet). The DWT can be considered a signal in a dyadic sub-band decomposition.

\subsection{Wavelet Packet}

Multi-resolution analysis can decompose the signal effectively in time-domain and frequency-domain, but because the scale function it uses changes in binary, the resolution in high frequency part of signal is poor. In order to improve the decomposing performance of high frequency part, the scale subspace $V_{j}$ and wavelet subspace $W_{j}$ are replaced by a new subspace $U_{j}^{2 n}$. Setting as $U_{j}^{0}=V_{j}, U_{j}^{1}=W_{j}, j \in \mathbf{Z}$, the orthogonal decomposition $V_{j+1}=V_{j} \oplus W_{j}$ can be replaced by

$$
U_{j+1}^{0}=U_{j}^{0} \oplus U_{j}^{1}
$$

Supposing function $u_{n}(t) \in U_{j}^{n}$ and function $u_{2 n}(t) \in U_{j}^{2 n}$, and then

$$
\left\{\begin{array}{l}
u_{2 n}(t)=\sqrt{2} \sum_{k \in \mathbf{Z}} h_{k} u_{n}(2 t-k) \\
u_{2 n+1}(t)=\sqrt{2} \sum_{k \in \mathbf{Z}} g_{k} u_{n}(2 t-k)
\end{array}\right.
$$

where $h_{k}$ is the filter coefficient of low frequency part and $g_{k}$ is the filter coefficient of high frequency part. When $n=0$, Eq. (9) is given by

$$
\left\{\begin{array}{l}
u_{0}(t)=\sqrt{2} \sum_{k \in Z} h_{k} \varphi(2 t-k) \\
u_{1}(t)=\sqrt{2} \sum_{k \in Z} g_{k} \varphi(2 t-k)
\end{array}\right.
$$

where $u_{0}(t)$ is called scaling function and $u_{1}(t)$ is called wavelet function. At this time, $\left\{u_{n}(t), n \in \mathbf{Z}_{+}\right\}$is the wavelet packet (WP) based on basis function $\varphi(t)$, its decomposing space is expressed by

$$
U_{j+1}^{n}=U_{j}^{2 n} \oplus U_{j}^{2 n+1}
$$

WP can make a multi-level partition for both low frequency part and high frequency part of signal at the same time, so it can make a more accurate analysis of signal. In the premise of meeting Heisenberg uncertainty principle, it can decompose signal into different frequency bands in line with the random time-frequency resolution, and project the time frequency component of signal into the corresponding orthogonal wavelet space to represent different frequency band.

\section{Proposed feedback structure}

For some broadband noise, the conventional de-noising method is to use nonlinear controllers, most popular one of which is neural network algorithm [14,15]. This way can predict and reduce chaotic noise, but useless for random noise, and the performance is not very well. In this paper, a new feedback structure based on WP using FXLMS algorithm is proposed to reduce the chaotic noise and random noise.

\subsection{Broadband noise prediction}

It is known that the WP can decompose signal into different frequency bands with the different resolution, every 
frequency band is limited and can be considered similarly as a narrowband signal. It means that every frequency signal is predictable, for explaining this phenomenon, the example of predicting logistic chaotic noise 1 and random noise are given as follows.
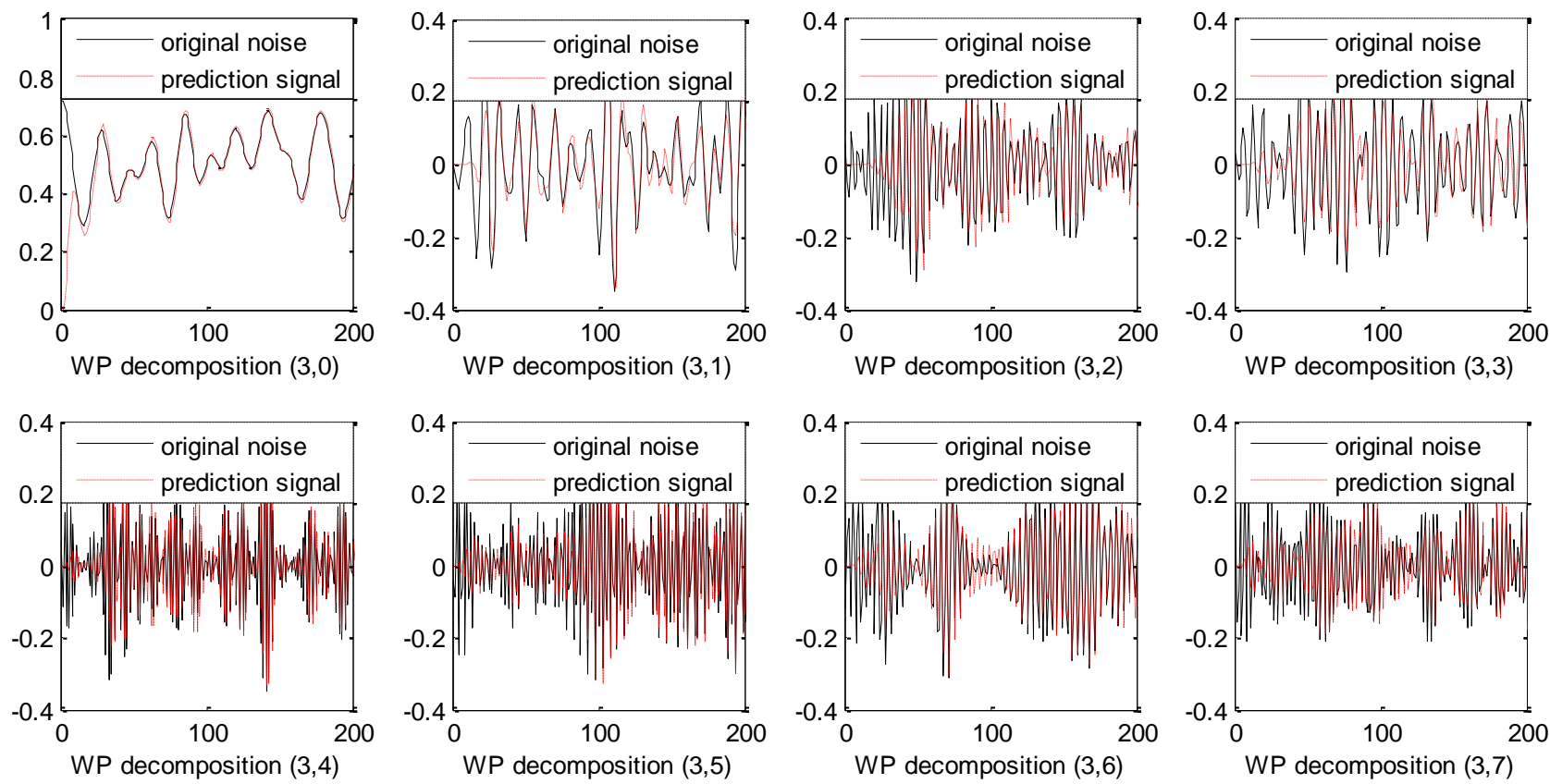

(a) Each part of logistic chaotic noise 1
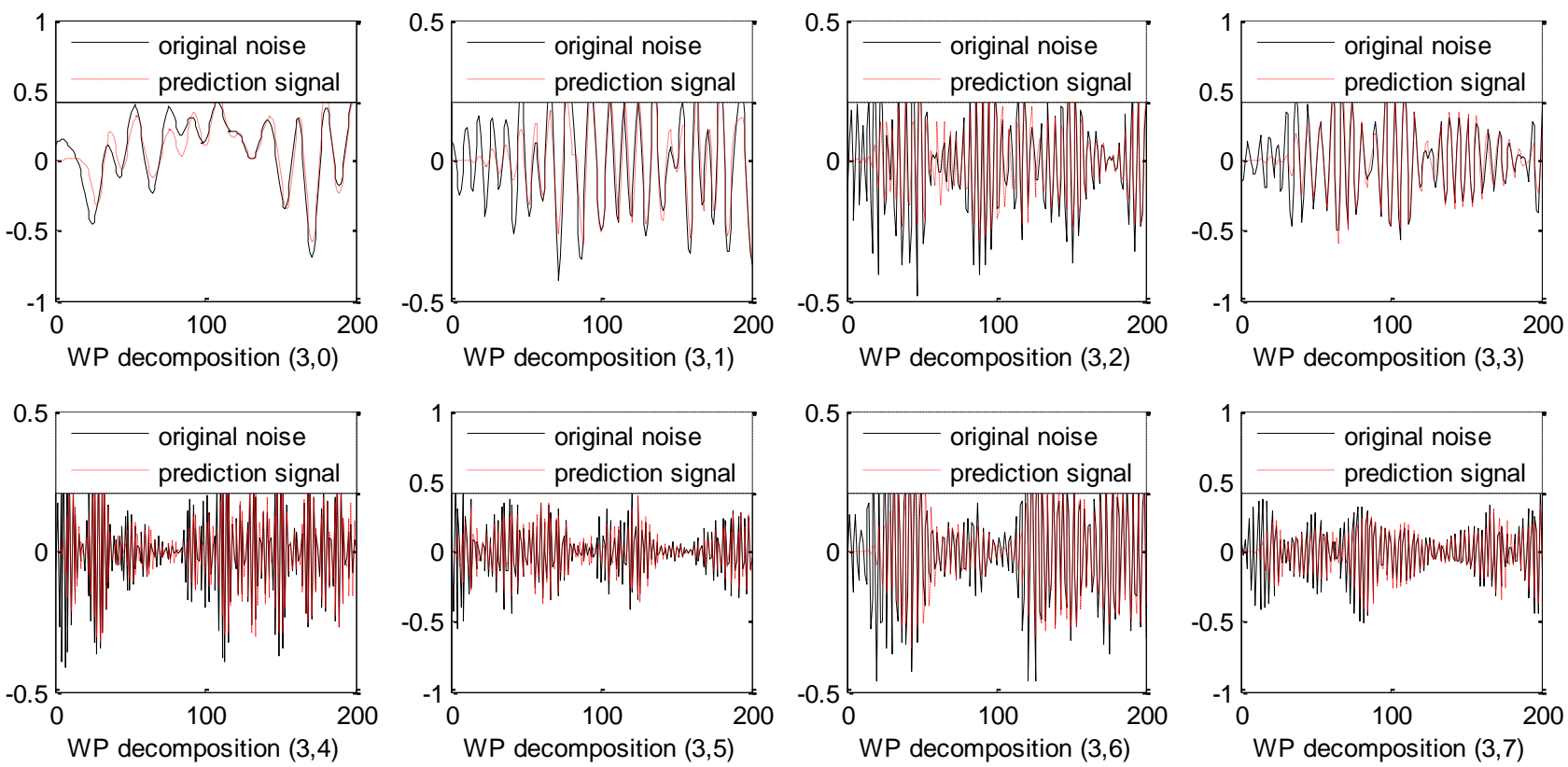

(b) Each part of random noise

Fig. 2 WP prediction for each part

Firstly, decomposing logistic chaotic noise 1 and random noise by WP 'dmey' with three layers, and then each part after decomposition is predicted by linear prediction technology like AR model. The each part of logistic chaotic noise 1 and random noise and their prediction are shown in Fig. 2 (a) and (b) respectively. It can be seen clearly that 
each part of the logistic chaotic noise 1 and random noise after WP decomposition is predicted well. This means that the integral chaotic noise and random noise cannot be predicted directly, but each part of them after WP decomposition can be predicted directly. This method provide a new idea to predict chaotic noise and random noise other than using nonlinear algorithms. The logistic chaotic noise 1 and random noise and their prediction using WP are shown in Fig. 3 (a) and (b). Through decomposition using WP and then prediction using linear model, the chaotic noise and random noise can also be predicted precisely.

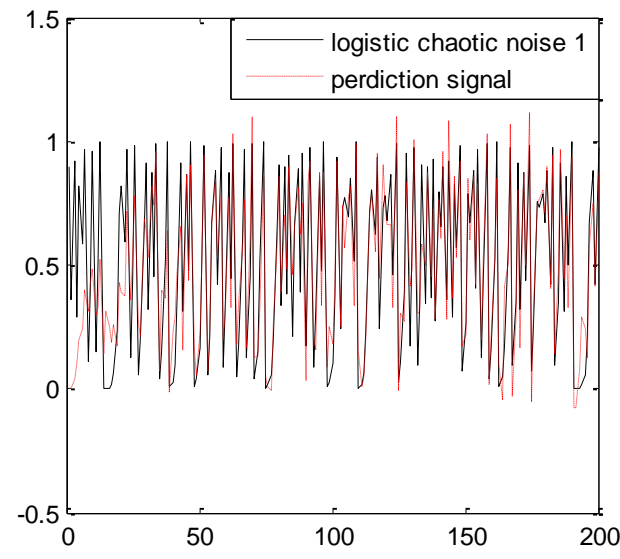

(a) Logistic chaotic noise 1

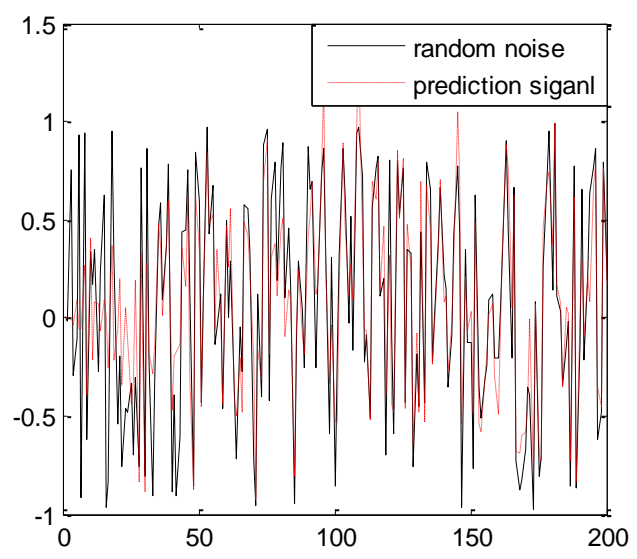

(b) Random noise

Fig. 3 The result of WP prediction

\subsection{Feedback ANC using WP}

In ANC system, supposing the primary noise is chaotic noise or random noise, which can be expressed by $p(n)$, secondary noise is $y(n)$, the error noise is given by

$$
e(n)=p(n)-y(n) * s(n)
$$

where the $s(n)$ is secondary path. Thus, the primary noise estimation can be expressed by

$$
d(n)=e(n)+y(n) * \hat{s}(n)
$$

$\hat{s}(n)$ is the estimated value of $s(n)$, If $\hat{s}(n)=s(n)$, then $d(n)=p(n)$. Because the $d(n)$ cannot be predicted directly using linear prediction technology, decomposing $d(n)$ firstly using WP. Supposing the decomposing layer is $K, d(n)$ can be decomposed $2^{K}$ components.

$$
d(n)=\sum_{i=1}^{2^{K}} \phi_{i}(n)
$$

It can be know above that the components of chaotic noise or random noise after WP can be predicted using linear technology. Therefore

$$
\phi_{i}(n)=\sum_{j=0}^{L_{i}} h_{i, j} \phi_{i}(n-1-j)+\varepsilon_{i}(n)
$$

where $\left\{\left\{h_{i, j}\right\}_{j=0}^{L_{i}}\right\}_{i=1}^{2^{K}}$ are the prediction coefficients and $\left\{\varepsilon_{i}(n)\right\}_{i=1}^{2^{K}}$ are the prediction error. In this paper, the $s(n)$ is non-minimum phase, so the Eq. (15) can be written as follow 


$$
\phi_{i}(n)=\sum_{m=0}^{M} \sum_{k=0}^{N_{i}} s_{m} w_{i, k} \phi_{i}(n-1-k-m)+\varepsilon_{i}(n)
$$

where $L_{i}=M+N_{i} . M$ and $\left\{s_{m}\right\}_{m=0}^{M}$ is the order and coefficients of secondary path $s(n) . N_{i}$ is the order of controller. Because $s(n)$ is known, we can find optimal linear prediction coefficients $\left\{\left\{h_{i, j}\right\}_{j=0}^{L_{i}}\right\}_{i=1}^{2^{K}}$ through calculating controller weights $\left\{\left\{w_{i, k}\right\}_{k=0}^{N_{i}}\right\}_{i=1}^{2^{K}}$. For simplifying the calculation, let $N=\left\{N_{i}\right\}_{i=1}^{2^{K}}$.

From the linear prediction process of primary noise $p(n)$, it can be known that the de-noising performance will better, if the secondary noise $y(n)$ is

$$
\begin{aligned}
y(n) & =\sum_{i=1}^{2^{K}} y_{i}(n) \\
& =\sum_{i=1}^{2^{K}} \sum_{k=0}^{N} w_{i, k} \phi_{i}(n-1-k)
\end{aligned}
$$

At this time, the ANC error $e(n)$ can be expressed by

$$
e(n)=\sum_{i=1}^{2^{K}} \varepsilon_{i}(n)
$$

If each part of original chaotic noise or random noise is predicted accurately, the error $e(n)$ reach its best result, so the key point is to find optimal controller weights $\left\{\left\{w_{i, k}\right\}_{k=0}^{N}\right\}_{i=1}^{2^{K}}$. Usually, these weights are updated by FXLMS algorithm

$$
\left\{\begin{array}{c}
w_{1}(n+1)=w_{1}(n)+\mu_{1} \varepsilon_{1}(n) \sum_{m=0}^{\hat{M}} \hat{s}_{m} \phi_{1}(n-m) \\
w_{2}(n+1)=w_{2}(n)+\mu_{2} \varepsilon_{2}(n) \sum_{m=0}^{\hat{M}} \hat{s}_{m} \phi_{2}(n-m) \\
\vdots \\
w_{2^{K}}(n+1)=w_{2^{K}}(n)+\mu_{2^{K}} \varepsilon_{2^{K}}(n) \sum_{m=0}^{\hat{M}} \hat{s}_{m} \phi_{2^{K}}(n-m)
\end{array}\right.
$$

where $w_{i}(n)=\left\{w_{i, k}\right\}_{k=0}^{N}, \hat{M}$ and $\left\{\hat{s}_{m}\right\}_{m=0}^{\hat{M}}$ are the order and coefficients of secondary path estimation $\hat{s}(n)$. $\left\{\varepsilon_{i}(n)\right\}_{i=1}^{2^{K}}$ cannot be obtained directly, so replacing them with their estimation $\left\{e_{i}(n)\right\}_{i=1}^{2^{K}}$ which are the result of $e(n)$ decomposed by WP. Then, the Eq. (19) is replaced by

$$
\left\{\begin{array}{c}
w_{1}(n+1)=w_{1}(n)+\mu_{1} e_{1}(n) \sum_{m=0}^{\hat{M}} \hat{s}_{m} \phi_{1}(n-m) \\
w_{2}(n+1)=w_{2}(n)+\mu_{2} e_{2}(n) \sum_{m=0}^{\hat{M}} \hat{s}_{m} \phi_{2}(n-m) \\
\vdots \\
w_{2^{K}}(n+1)=w_{2^{K}}(n)+\mu_{2^{K}} e_{2^{K}}(n) \sum_{m=0}^{\hat{M}} \hat{s}_{m} \phi_{2^{K}}(n-m) \\
e(n)=\sum_{i=1}^{2^{K}} e_{i}(n)
\end{array}\right.
$$

After analysis above, the proposed feedback ANC using WPFXLMS algorithm is shown in Fig. 4. The WP used 
in primary noise estimation $d(n)$ is the same as the WP used in error $e(n)$, so $\left\{\phi_{i}(n)\right\}_{i=1}^{2^{K}}$ and $\left\{e_{i}(n)\right\}_{i=1}^{2^{K}}$ have the same frequency band. By using WP, each part of chaotic noise or random noise after WP decomposition is controlled independently, because each part can be seen as a narrowband noise signal which is easier to reduce, the control process and de-noising performance are improved better.

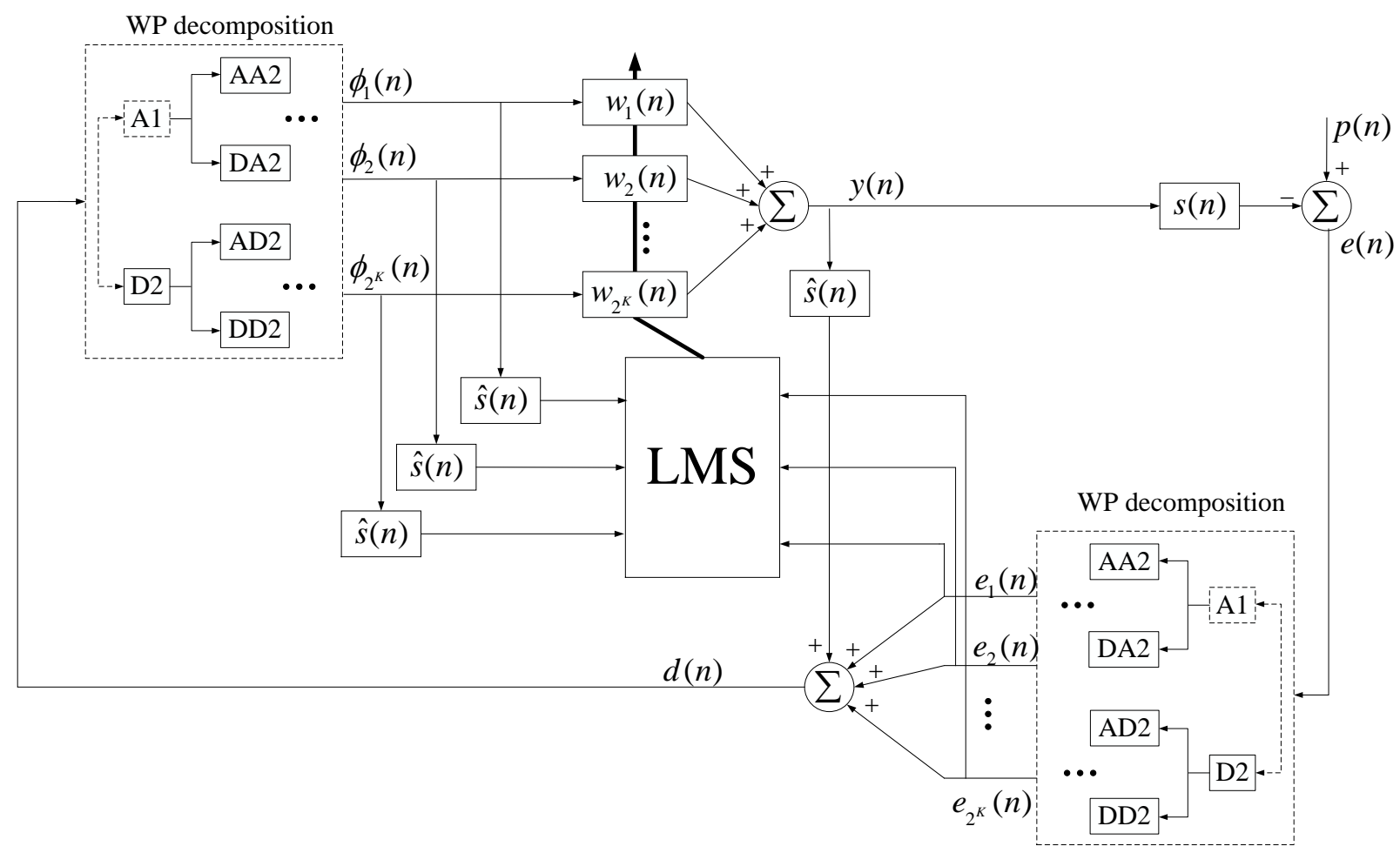

Fig. 4 Proposed feedback ANC using WPFXLMS algorithm

\subsection{Computational complexity analysis}

Computational complexity is an important index to evaluate the effectiveness of the ANC algorithms, low computational complexity requires less resources and computation time to ensure real-time signal processing in an ANC system. Therefore, for the purpose of practical implementation, a low computational complexity ANC algorithm is always in demand. In this section, the computational complexity of the proposed WPFXLMS algorithm is evaluated and compared with that of FXLMS algorithm and FSLMS algorithm. Providing that the memory size of all involved algorithms is $N$, the order of secondary path estimation is $\hat{M}$, the WP decomposition layer is $K$. Generally speaking, there are three procedures in feedback ANC system:

(a) Producing the secondary noise $y(n)$

Multiplications: $2^{K}(N+1)$

Additions: $2^{K}(N+1)$

(b) Updating the adaptive filter coefficients $\left\{\left\{w_{i, k}\right\}_{k=0}^{N}\right\}_{i=1}^{2^{K}}$

Multiplications: $2^{K}(N+1)(\hat{M}+1)$

Additions: $2^{K}(N+1) \hat{M}$

(c) Calculating primary noise estimation $d(n)$

Multiplications: $\hat{M}+1$ 
Additions: $\hat{M}+1$

Other than the computation above, the WPFXLMS also needs two times wavelet packet transform, which is Multiplications: $2^{K}(N+1)$

Additions: $2^{K}(N+1)$

The comparison of computational complexity of FXLMS algorithm, FSLMS algorithm and WPFXLMS algorithm is listed in Table I. It is clear that FXLMS algorithm needs lest computational complexity among them, but FXLMS algorithm is linear controlling algorithm which is invalid for nonlinear noise and broadband noise. $P$ is the order of FSLMS algorithm, when $P=3$, the computational complexity of FSLMS algorithm is less than that of WPFXLMS algorithm, and when $P=4$, the computational complexity of FSLMS algorithm is more than that of WPFXLMS algorithm. Moreover, FSLMS algorithm also needs calculate sine and cosine computation.

Table I

Computational complexity comparison of FXLMS algorithm, FSLMS algorithm and WPFXLMS algorithm

\begin{tabular}{|c|c|c|c|c|}
\hline Algorithm & FXLMS & \multicolumn{2}{|c|}{ FSLMS } & WPFXLMS \\
\hline Multiplications & $(N+1)(\hat{M}+2)+\hat{M}+1$ & \multicolumn{2}{|c|}{$(2 P+1)(N+1)(\hat{M}+2)+\hat{M}+1$} & $2^{K}(N+1)(\hat{M}+3)+\hat{M}+1$ \\
\hline Additions & $(N+1)(\hat{M}+1)+\hat{M}+1$ & \multicolumn{2}{|c|}{$(2 P+1)(N+1)(\hat{M}+1)+\hat{M}+1$} & $2^{K}(N+1)(\hat{M}+2)+\hat{M}+1$ \\
\hline \multicolumn{2}{|c|}{$N=50, \hat{M}=15$} & $P=3$ & $P=4$ & $K=3$ \\
\hline Multiplications & 883 & 6085 & 7819 & 7360 \\
\hline Additions & 832 & 5728 & 7360 & 6952 \\
\hline
\end{tabular}

\section{Simulation studies}

In this section, numerous simulation experiments are conducted to evaluate the performance of the proposed WPFXLMS algorithm by processing different types of logistic chaotic noise and random noise. The wavelet used in the simulation is 'dmey' and decomposing level is $K=3\left(2^{3}=8\right.$ parts). The time domain characteristic of the 'dmey' wavelet is shown in Fig. 5.

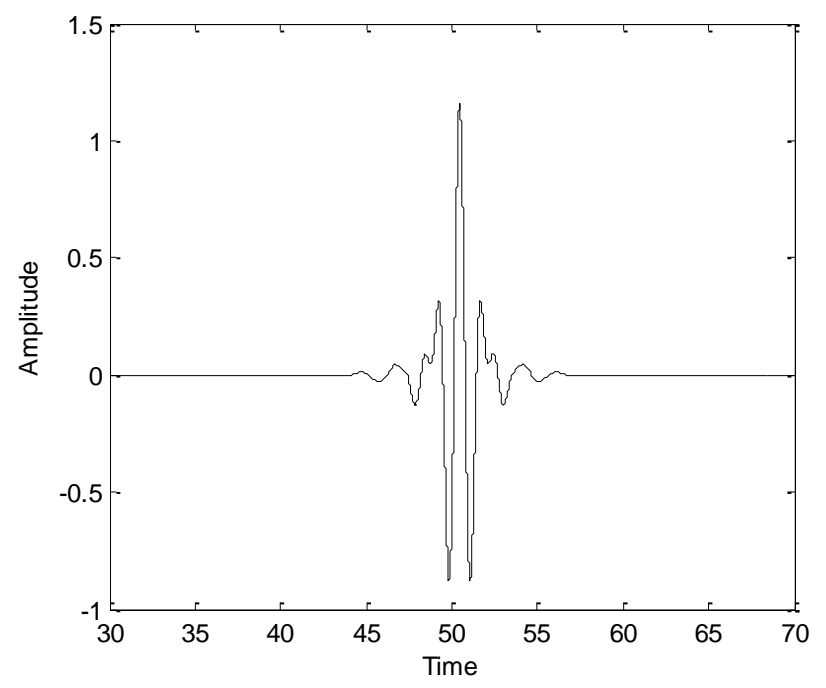

Fig. 5 The 'dmey' wavelet

In all experiments, the secondary path is considered to be an FIR filter with non-minimum phase [23-25], whose transfer function is given by

$$
S(z)=z^{-2}+1.5 z^{-3}-z^{-4}
$$


The secondary path is estimated off-line with a white Gaussian noise whose variance is one by LMS algorithm, the order is $\hat{M}=15$. Forty independent trials are conducted to calculate the mean behaviors of them.

For comparison, FXLMS algorithm and FSLMS algorithm are involved. The order of the functional expansion of FSLMS algorithm is considered as $P=3$ and $P=4$. In all simulation experiments, the step sizes for the FXLMS algorithm, FSLMS algorithm and proposed WPFXLMS algorithm are totally chosen to be $\mu=0.0001$, and memory size is 50 [15].

\subsection{Experiment I}

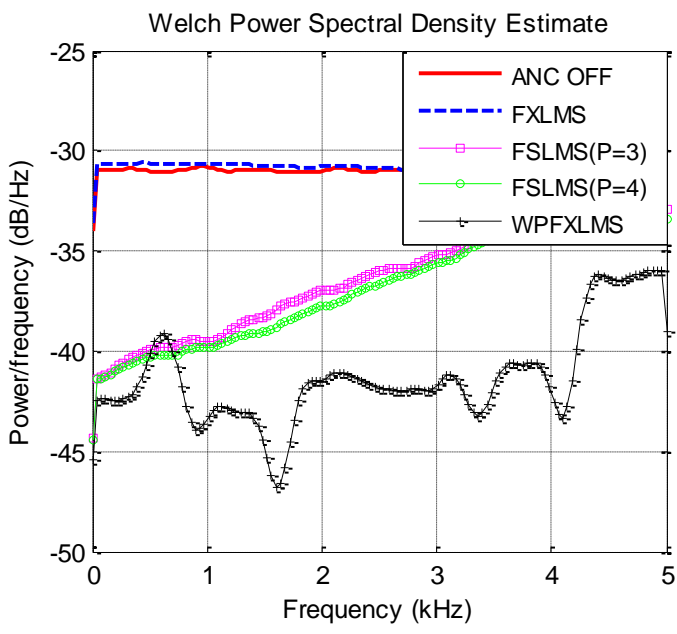

(a) PSD

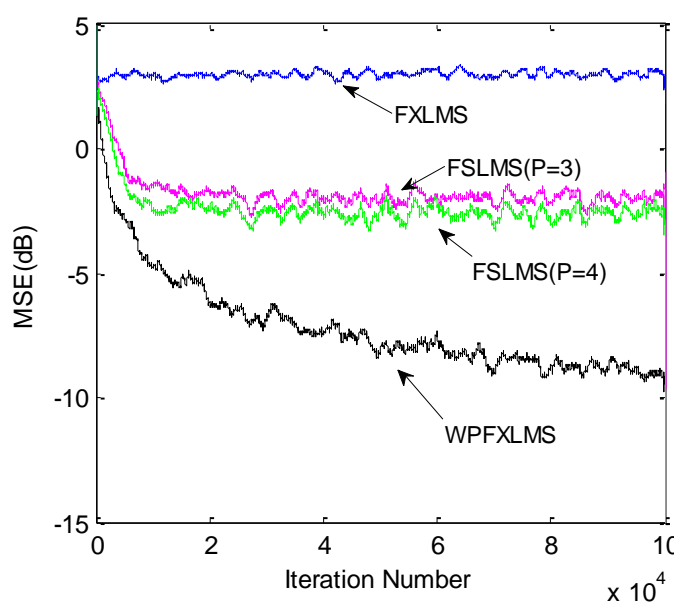

(b) MSE learning curves

Fig. 6 The result of reducing logistic chaotic noise 1

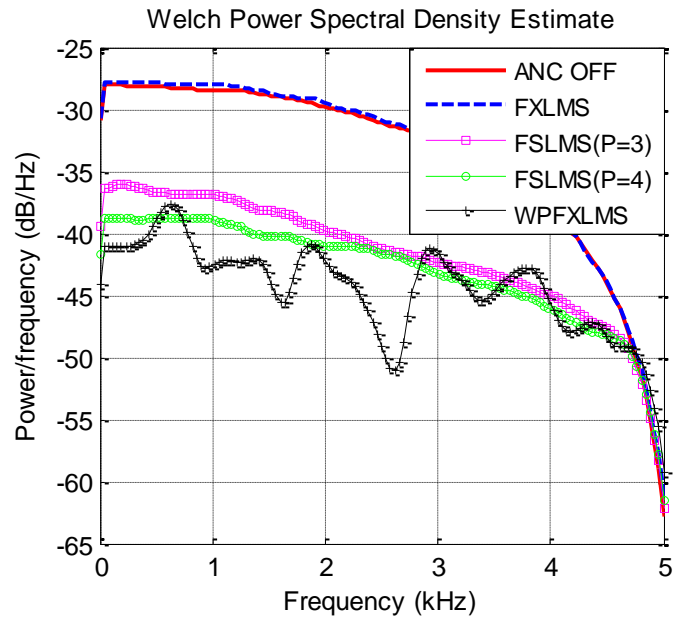

(a) PSD

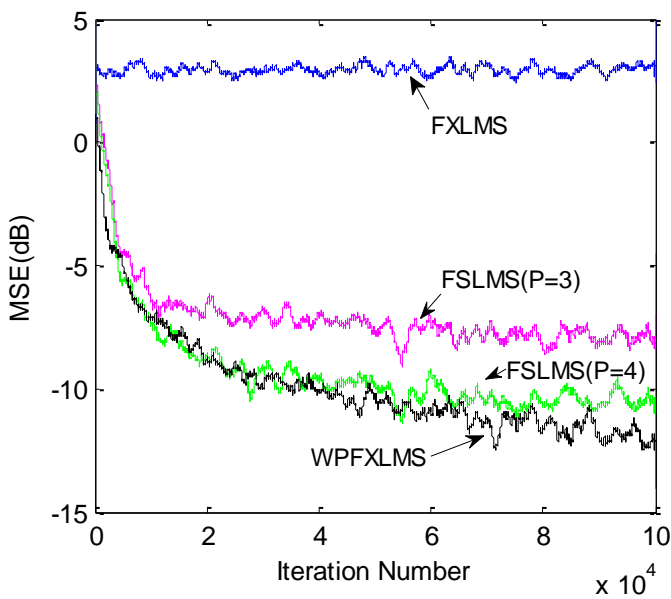

(b) MSE learning curves

Fig. 7 The result of reducing logistic chaotic noise 2

In this part, the primary noise is logistic chaotic noise $Q$ where $(Q=1,2)$, and they are all normalized to have zero mean and signal power is 2 . The power spectral density (PSD) of logistic chaotic noise $Q$ and the mean square error (MSE) results of FXLMS algorithm, third order and fourth order FSLMS algorithm, proposed WPFXLMS algorithm are plotted in Fig. 6, Fig. 7. It is clear that FXLMS algorithm is almost invalid to reduce the logistic chaotic noise, and the FSLMS algorithm can reduce this noise, the fourth order performs better than third order. However, the proposed WPFXLMS algorithm performs best among them. With the increase of $Q$, the frequency band of logistic chaotic noise is narrowed down, and the broadband property presents weaker, so the performance 
of WPFXLMS algorithm is close to that of FSLMS algorithm. This can prove that the proposed WPFXLMS algorithm has much more advantages to reduce broadband noise, but less advantages to reduce narrowband noise.

\subsection{Experiment II}

In this experiment, the primary noise is chosen as Henon chaotic noise which is shown in Eq. (3), it is also normalized to have zero mean and signal power is 2. It can be seen from the PSD of Fig. 8 (a), the Henon chaotic noise is also a broadband signal. The Fig. 8 (b) shows the MSE performances of FXLMS algorithm, FSLMS( $P=3$ ) and FSLMS $(P=4)$ algorithms and WPFXLMS algorithm, FXLMS algorithm still does not work at all for this chaotic noise, when $P=3$ and $P=4$, the FSLMS algorithm performs similar on noise reduction. Proposed WPFXLMS algorithm still outperforms the other two algorithms.

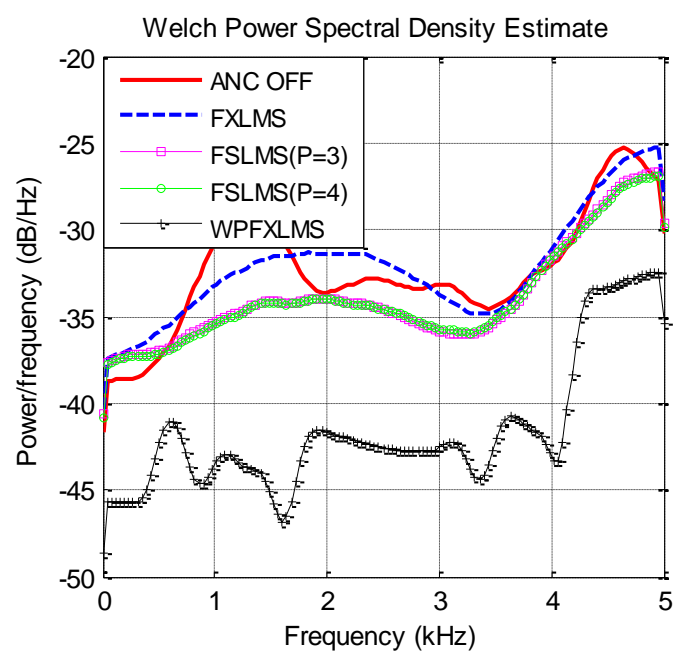

(a) PSD

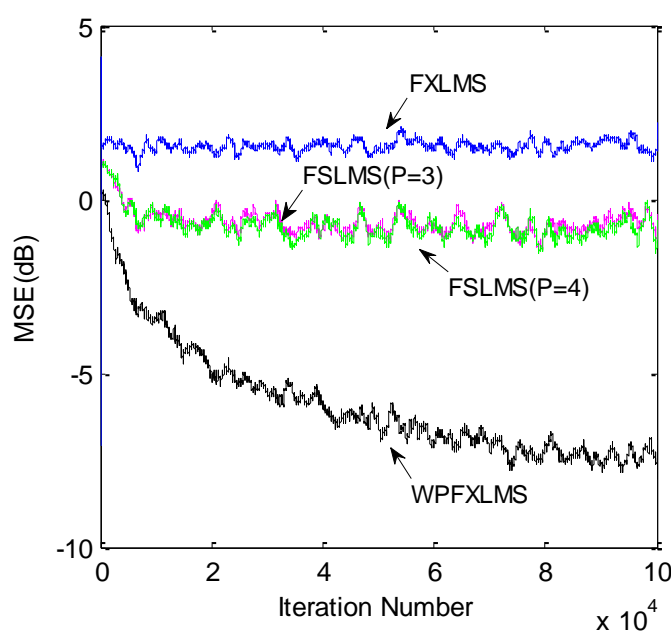

(b) MSE learning curves

Fig. 8 The result of reducing Henon chaotic noise

\subsection{Experiment III}

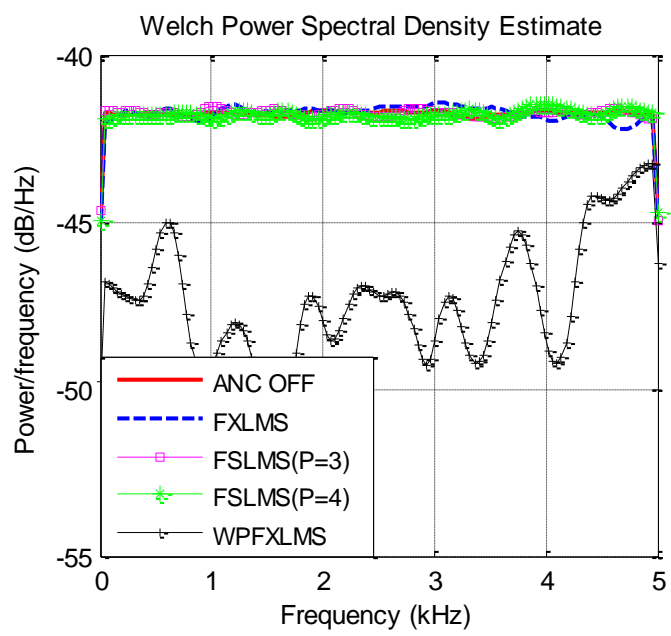

(a) PSD

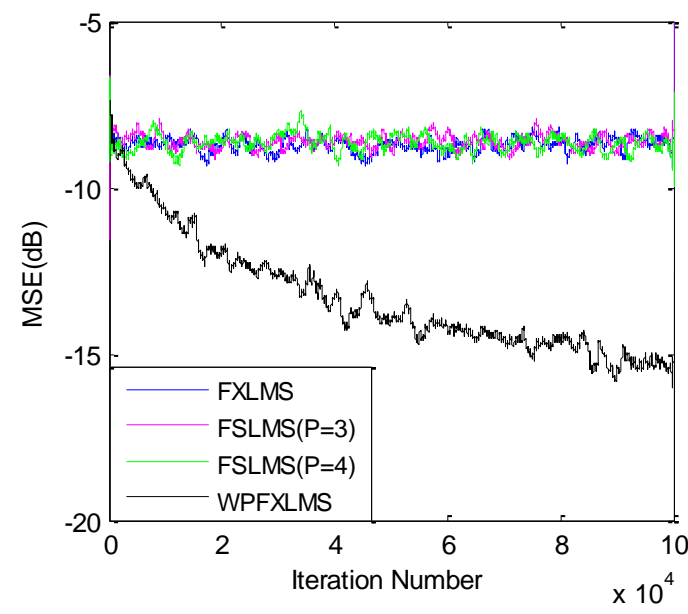

(b) MSE learning curves

Fig. 9 the result of reducing random noise

In this experiment, the primary noise is chosen as a random noise which is shown in Eq. (4). The result of each 
algorithm to reduce this noise is shown in Fig. 9, where (a) is the PSD plot and (b) is the MSE plot. From the plots, it is clear that FXLMS algorithm and FSLMS algorithm cannot reduce random noise at all, but the proposed WPFXLMS algorithm still work very well.

\subsection{Experiment IV}

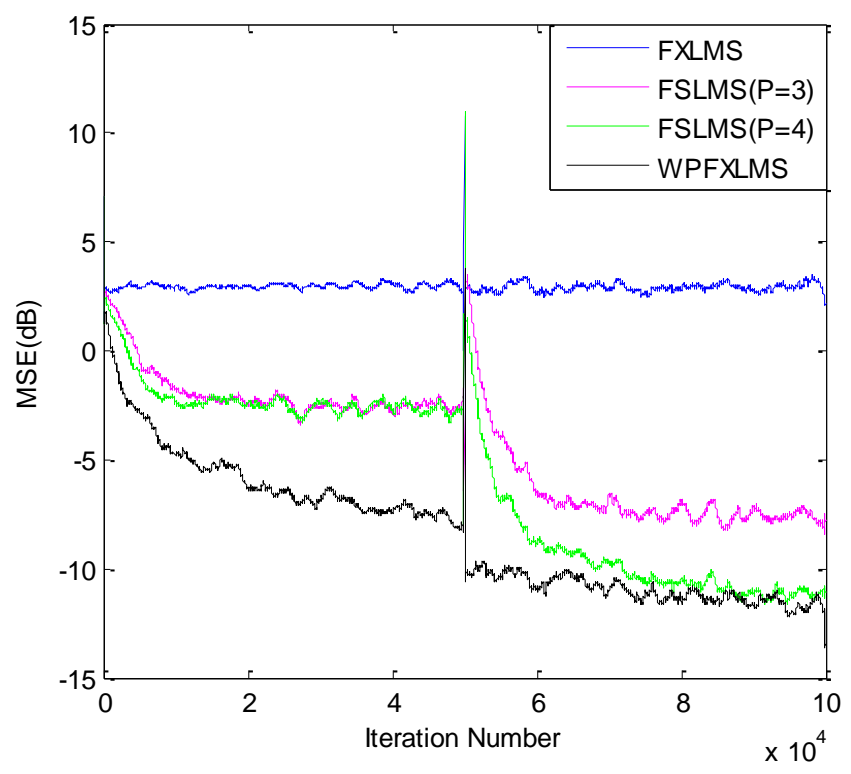

Fig. 10 The result of reducing changed primary noise

In this experiment, the adaptation and tracking capability of the proposed WPFXLMS algorithm is checked by changing the primary noise type. In the first half part, from iteration 1 to 50000, the primary noise is logistic chaotic noise 1 , and after iteration 50000 , the primary noise changes to logistic chaotic noise 2 . In the whole process, the noise is normalized to have zero mean and signal power is 2 . The result of each algorithm to reduce this noise is shown in Fig. 10, in which we can know that the tracking capability of proposed WPFXLMS is best, it almost does not need to spend time to adjust its de-noising process again when this change happens. This is mainly because that the advantage of WP decomposition to deal with non-stationary signal.

\section{Conclusions}

In this paper, we propose a new feedback ANC scheme for broadband noise like chaotic noise and random noise, it is wavelet packet FXLMS (WPFXLMS) algorithm. By analyzing the feedback ANC system with non-minimum phase secondary path, it can be know that each part of the broadband noise after WP decomposition can be predicted directly using linear prediction technology. For evaluating the performance of the proposed WPFXLMS algorithm, many simulations are conducted, from these simulations it can be found that proposed WPFXLMS algorithm outperforms FXLMS and FSLMS algorithms on broadband noise cancellation, especially the random noise. For some non-stationary change, the proposed WPFXLMS algorithm also presents best adaptation and tracking capability. Different simulations have demonstrated the effectiveness of proposed WPFXLMS algorithm.

\section{Acknowledgement}

This work was partially supported by National Science Foundation of PR China (Grant 1171183, 61471140), Aerospace Support Fund (01320214) and Natural Scientific Research Innovation Foundation in Harbin Institute of Technology (HIT.NSRIF201508954). 


\section{Reference}

[1] Eberhard Hansler, Gerhard Schmidt. Acoustic Echo and Noise Control: A Practical Approach. Wiley; 2004.

[2] Kuo SM, Morgan DR. Active noise control: a tutorial review, Proceedings of IEEE 1999; 87 (6) : 943-973.

[3] Kuo SM, Morgan DR. Active Noise Control Systems: Algorithms and DSP Implementations. New York: Wiley; 1996.

[4] Elliott SJ, Sutton TJ. Performance of feedforward and feedback systems for active control. IEEE Trans. Speech and Audio Processing 1996; 4 (3): 214-223.

[5] Zhang L, Wu L, Qiu X. An intuitive approach for feedback active noise controller design. Appl. Acoust. 2013; 74(1): 160-168.

[6] Kuo SM, Song Y, Gong Y. A robust hybrid feedback active noise cancellation headset. IEEE Trans Speech Audio Process 2005; 13(4): 607-617.

[7] Ganguly A, Panahi I, Dufour F. Exploring feedback active noise control with ambisonics. IEEE Circuits and Systems Conference (DCAS) 2015; 1-4.

[8] Lifu Wu, Xiaojun Qiu, Yecai Guo. A simplified adaptive feedback active noise control system. Appl. Acoust. 2014; 81: 40-46.

[9] Bouchard M, Paillard B. An alternative feedback structure for the adaptive control of periodic and time varying periodic disturbances. Sound and Vibration1998; 210 (4): 517-527.

[10] Schumacher T, Kruger H, Jeub M, Vary P, Beaugeant C, Active noise control in headsets - a new approach for broadband feedback ANC, IEEE Trans. Signal Process 2011; 978 (1) : 4577-5039.

[11] Shibalik Mohapatra, Asutosh Kar. An Advanced Feedback Filtered-x Least Mean Square Algorithm for Wideband Noise Removal. IEEE ECTI-CON 2015; 1-5.

[12] Strauch P, Mulgrew B. Active control of nonlinear noise processes in a linear duct, IEEE Trans Signal Process 1998; 46: 2404-2412.

[13] Das DP, Moreau DJ, Cazzolato BS. A nonlinear active noise control algorithm for virtual microphones controlling chaotic noise, J. Acoust. Soc. Am. 2012; 132 (2): 779-788.

[14] Behera SK, Das DP, Subudhi B. Functional link artificial neural network applied to active noise control of a mixture of tonal and chaotic noise. Appl. Soft Comput 2014; 23: 51-60.

[15] Behera SB, Das DP, Rout NK. Nonlinear feedback active noise control for broadband chaotic noise, Appl. Soft Comput. 2014; 15: 80-87.

[16] Hilton M. Wavelet and wavelet packet compression of electrocardiograms. IEEE Trans. Biomed. Eng. 1997; 44 (5): 394-402.

[17] Brechet L, Lucas MF, Doncarli C, Farina D. Compression of biomedical signals with mother wavelet optimization and best-basis wavelet packet selection. IEEE Trans. Biomedical Engineering 2007; 54 (12): 2186-2192.

[18] Hosur S, Tewfik AH. Wavelet transform domain LMS algorithm. IEEE ICASSP1993; 3: 508-510.

[19] Attallah S, Najim M. A fast wavelet transform-domain LMS algorithm. IEEE ICASSP 1996; 3:1343-1346.

[20] Qiu Z, Lee C-M, Xu ZH, Sui L.N. A multi-resolution filtered-x LMS algorithm based on discrete wavelet transform for active noise control. Mechanical Systems and Signal Processing 2016; 66: 458-469.

[21] Zu-Ren Feng, Qing Zhou, Jun Zhang, Ping Jiang, Xue-Wen Yang. A Target Guided Subband Filter for Acoustic Event Detection in Noisy Environments Using Wavelet Packets. IEEE/ACM Tans. Audio, Speech, and Language Processing 2015; 23 (2): 361-372. 
[22] Li Y, Zhang L, Li B, Yan G. The comparison study of wavelet and wavelet packet analysis in the de-noising of EEG test signal. ITME 7th 2017; 274-277.

[23] Haiquan Zhao, Xiangping Zeng, Jiashu Zhang. Adaptive reduced feedback FLNN filter for active control of nonlinear noise processes, Signal Processing 2010; 90 (3): 834-847.

[24] Vinal Patel, Nithin VG. Nonlinear active noise control using spline adaptive filters, Appl. Acoust. 2015; 93 : 38-43.

[25] Nithin VG, Ganapati Panda. A robust filtered-s LMS algorithm for nonlinear active noise control, Appl. Acoust. 2012; 73: 836-841. 